\title{
Cavity-Enhanced Vernier Spectroscopy with a Chip-Scale Mid-Infrared Frequency Comb
}

\author{
Lukasz A. Sterczewski, ${ }^{*}, \uparrow, \uparrow, \|$ Tzu-Ling Chen, ${ }^{\ddagger}, \|$ Douglas C. Ober, ${ }^{\ddagger}, \dagger$ Charles R. Markus, ${ }^{\ddagger}$ \\ Chadwick L. Canedy,,$^{\S}$ Igor Vurgaftman, ${ }^{\S}$ Clifford Frez, ${ }^{\dagger}$ Jerry R. Meyer, ${ }^{\S}$ Mitchio Okumura, ${ }^{*}, \ddagger$ \\ and Mahmood Bagheri*,† \\ $\dagger$ Jet Propulsion Laboratory, California Institute of Technology, Pasadena, CA 91109, USA \\ $\ddagger$ Division of Chemistry and Chemical Engineering, California Institute of Technology, Pasadena, \\ CA, 91125, USA \\ \Faculty of Electronics, Photonics and Microsystems, Wroclaw University of Science and \\ Technology, Wroclaw 50370, Poland \\ $\S$ Optical Sciences Division, Naval Research Laboratory, Washington, DC 20375, USA \\ $\|$ These authors contributed equally to this work. \\ E-mail: lukasz.sterczewski@pwr.edu.pl; mo@caltech.edu; mahmood.bagheri@jpl.nasa.gov
}

\begin{abstract}
Chip-scale optical frequency combs can provide broadband spectroscopy for diagnosing complex organic molecules. They are also promising as miniaturized laser spectrometers in applications ranging from atmospheric chemistry to geological science and the search for extraterrestrial life. While optical cavities are commonly used to boost sensitivity, it is challenging to realize a compact cavityenhanced comb-based spectrometer. Here, we apply the Vernier technique to free-running operation of an interband cascade laser frequency comb in a simple linear geometry that performs cavityenhanced chemical sensing. A centimeter-scale high-finesse cavity simultaneously provides selective mode filtering and enhancement of the path length to 30 meters. As a proof-of-concept, we sense transient open-path releases of ppm-level difluoroethane with $2 \mathrm{~ms}$ temporal resolution over a $1 \mathrm{THz}$ optical bandwidth centered at $3.64 \mu \mathrm{m}$.
\end{abstract}

\section{Abbreviations}

OFC,ICL,QCL,DCS,VS,ISO,PZT

\section{Keywords}

interband cascade laser, frequency comb, midinfrared, Vernier spectroscopy, cavity-enhanced
Optical frequency combs (OFCs) ${ }^{112}$ have advanced the maturation of broadband high-resolution laser spectroscopy for molecular sensing. The discrete pattern of sharp equidistant lines spanning broad spectral bandwidth that can be metrologically traced to primary frequency standards has advanced fundamental science ${ }^{3}$ and revolutionized the fields of precision optical metrology 4 and chemical sensing. ${ }^{5}$ Miniaturization has transformed bulky laboratory-grade sources into compact emitters operating across diverse spectral regions, which allows exploitation of the OFC advantages in portable scenarios. $\frac{66}{8}$ Electrically-pumped chip-scale $\mathrm{OFC}$ sources $^{7}$ based on quantum cascade lasers (QCLs) ${ }^{9}$ and interband cascade lasers $(\mathrm{ICLs})^{10} \sqrt{12}$ are of particular interest due to fundamental molecular absorption bands in the midwaveinfrared (mid-IR) spectral region.

A primary factor limiting the compactness and complexity of broadband OFC spectroscopy has been resolution of the individual comb teeth. While this can be accomplished using dispersive Fouriertransform methods, $\frac{13}{13}$ a popular alternative is dualcomb spectroscopy (DCS).14 DCS is a moderesolved Fourier-transform technique that converts optical information into an electrical signal via multi-heterodyne beating. 14 The DCS architecture requires no moving parts and can provide high tem- 
poral resolution on the order of nano- -15 to microseconds. $\frac{17]}{17}$ Nonetheless, experimental challenges include the requirements for high data throughput, microwave-compatible digitizers, and fast photodetection. Consequently, the hardware resources needed to acquire and process DCS signals (interferograms) can burden the instrument power budget and increase overall complexity.

Furthermore, miniaturization of the instrument can be compromised when high sensitivity $(\mathrm{ppm} / \mathrm{ppb})$ is needed.16 Such measurements typically require lenghtening of the optical path via a multi-pass cell 18 or high-finesse optical cav-

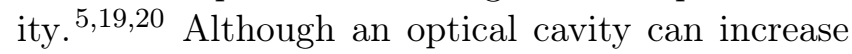
the effective path length to kilometers, the resonance condition must be also satisfied, which in turn requires locking of the comb and cavity. Finally, DCS strictly requires a mutual coherence time greater than the electrical interferogram repetition rate, which is typically generated by hardware lock loops or shared-cavity dual-comb lasing. 21 Even then, digital phase post-correction techniques 22 are often needed to accurately recover the mode-resolved spectroscopic information.

An alternative technique that perfectly matches the requirements for compact chip-based OFC spectrometry and circumvents many of the above issues is Vernier spectroscopy (VS). ${ }^{25}$ At its heart is a tunable high-finesse optical cavity (scanning Fabry-Pérot interferometer) that simultaneously filters the optical spectrum and enhances the path length. Since VS needs only a single comb source (unlike DCS), the system can operate entirely in free-running mode for significant reduction of the size and complexity. The VS optical cavity functions by providing a secondary scale with respect to the mode spacing of the comb, as in the mechanical Vernier caliper invented in 1631 by Pierre Vernier. ${ }^{26}$ Because the two scales are mismatched, it is possible to get coincidence between one line from each scale, which in this case corresponds to one comb tooth and one cavity mode (Fig. 1 1a). When applied to tabletop OFC sources, 27 29 VS has attained impressive temporal resolution and precision. However, the comb's low repetition rate allowed only groups of modes to be filtered instead of single teeth. Also, to allow for very broad optical bandwidth an optical grating mounted on a moving galvo scanner, and synchronized with the piezoelectric transducer (PZT), was needed to disperse higher Vernier orders and prevent groups of comb lines at different wavelengths from reaching the photodetector simultaneously.

In this work, we have used the VS technique to resolve single comb lines in a simple linear geometry. No moving gratings or control loops are needed for spectrally-sparse (yet tunable) semiconductor microcombs, because the Vernier orders can be separated by more than the comb bandwidth while the Vernier cavity filter resolution is much finer than the comb line spacing. Chip-based VS can be seen as complementary to other direct frequency comb techniques like virtual imaging phase array (VIPA) ${ }^{30}$ spectroscopy, but with the advantage of requiring only a slow single-pixel photodetector rather than a bulky and more expensive array or camera. Beyond the mid-IR, this versatile technique may also be applied to micro-comb sensing systems that cover other challenging spectral regions like the long-wave infrared (LWIR) and even terahertz. 31

Figure 1 illustrates the VS principle (a) and experimental setup (b). For this prototype, the entire optical layout, including the ICL comb, is contained within a $1.5^{\prime} \times 2^{\prime}$ area. The frequency comb is a GaSb-based ICL active structure ${ }^{32}$ processed into a narrow ridge divided into gain and saturable absorber sections ${ }^{10}$ similar to that reported in Ref. ${ }^{33}$ The ICL is biased in a low-phase-noise regime with $<1 \mathrm{~W}$ of electrical power consumption, and emits a 1-THz-spanning spectrum centered at $\nu_{c}=2750 \mathrm{~cm}^{-1}\left(\lambda_{c}=3.636 \mu \mathrm{m}\right)$. On careful alignment of a Faraday optical isolator (ISO) and collimation lens, the intermode (repetition rate) beat note centered at $f_{\text {rep }}=9.6991 \mathrm{GHz}$ preserves a feedback-free sub-kHz RF linewidth accompanied by $\sim 300 \mathrm{kHz}$ optical line width (estimated from DCS experiments). Narrow optical lines are critical for efficient coupling into the cavity with mode width $\Gamma=1.59 \mathrm{MHz}$, since a multi-MHz linewidth broadened by feedback-induced phase noise would effectively preclude mode-resolved measurements and lower the optical transmission by orders of magnitude.

The open-path Vernier cavity shown in Fig. 11 consists of two identical concave mirrors (6 meters radius of curvature) formed by highly-reflective coatings deposited on polished silicon substrates (Los Gatos Research Inc.). The cavity finesse $\mathcal{F}$ was evaluated from the mirror reflectivity data, and independently by a cavity ringdown measurement performed with a single mode laser whose optical frequency is matched to the ICL comb. The ringdown time of $\tau \approx 100$ ns yields $\mathcal{F} \approx 3050$ and 

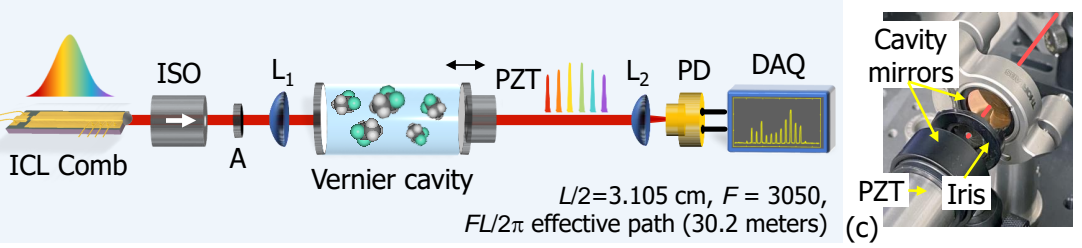

Figure 1: (a) Principle of mode-resolved VS, where the dashed lines are cavity modes with spacing and positions that change as the piezo actuator is scanned. Coincidence occurs for only one comb line at a time. Note that the cavity FSR is half the comb repetition rate, therefore every other cavity mode sequentially filters the comb spectrum. (b) Experimental setup of the chip-based VS. (c) Photo of the open-path Vernier cavity.

$R_{1,2}=99.897 \%$, which is consistent with the specified mirror reflectivity of $\approx 99.9 \%$ at this wavelength. One of the cavity mirrors is mounted on a piezoelectric transducer (PZT) with maximum displacement $\lambda_{\max } \approx 4 \mu \mathrm{m}$ that allows scanning the comb spectrum at sub-kHz rate over two Vernier free spectral ranges (FSRs). The scan rate is limited by the inertia of the mirror/piezo assembly and the photon lifetime in the FP resonator. 27

For efficient coupling into the cavity, the input beam is mode-matched by a lens $\left(\mathrm{L}_{1}, f=\right.$ $500 \mathrm{~mm}$ ) that focuses to a point between the mirrors spaced nominally by $L / 2 \approx 3.105 \mathrm{~cm}$ (beam waist $\sim 0.47 \mathrm{~mm})$. Since the corresponding cavity $\mathrm{FSR}_{c}=4.830 \mathrm{GHz}$ is approximately half the comb repetition rate, every other cavity mode participates in the scanned mode filtering. The cavity roundtrip length $L$ was determined by carefully detuning from the perfect-match condition $L_{0} / 2=c / f_{\text {rep }}=3.093 \mathrm{~cm}$, such that the measured photodetector signal extended over at least twice the comb's 10-dB bandwidth of $1 \mathrm{THz}$ when the PZT moved by $\lambda_{c} / 2$. This allowed for slight drifts of the comb offset frequency $f_{0}$ that appeared as a shift of the time-of-arrival (TOA) for the Vernier spectra. The relative comb-cavity mismatch $\epsilon=$ $\Delta L / L_{0}=2 \cdot 10^{-3}$ translates to $\Delta L \approx 123.7 \mu \mathrm{m}$, and yields Vernier $\mathrm{FSR}_{v}=c / \Delta L=2.425 \mathrm{THz}$, which represents the maximum unaliased optical coverage. For spectroscopy, the roundtrip length $L$ is enhanced ${ }^{27}$ by $\mathcal{F} / 2 \pi \approx 486$ to an effective path length $L_{\text {eff }}=30.2 \mathrm{~m}$.

A ZnSe objective $\left(\mathrm{L}_{2}\right)$ focused the cavityfiltered light onto a four-stage thermoelectricallycooled $\mathrm{HgCdTe}$ photodetector (PD) with a transimpedance preamplifier (VIGO PVI-4TE-3.4). Given the current responsivity of the $\operatorname{PD} \Re_{I}\left(\lambda_{c}\right) \approx$ $80 \mathrm{~mA} / \mathrm{W}$ at the relevant wavelength, and the transmimpedance gain $K_{i} \approx 200 \mathrm{kV} / \mathrm{A}$, we de- rive a voltage responsivity of $16 \mathrm{mV} / \mu \mathrm{W}$. The $\mathrm{PD}$ electrical signal was acquired by a 12-bit digitizer (DAQ, Zurich UHFLI) operated in oversampling mode $(7 \mathrm{MS} / \mathrm{s})$. From the average peak signal level of tens of $\mathrm{mV}$, we estimate a $\mu \mathrm{W}$-level optical power per comb line. This is $<10 \%$ of the light emitted at the ICL facet $(8 \mathrm{~mW}$ distributed over $\sim 100$ lines) because the throughput is severely limited by apertures in the beam path (A) used to improve the slightly elliptical beam profile that would otherwise excite higher order cavity modes. Without beam shaping, the photodetector signal from higher order cavity modes would overlap the fundamental and cause spectral clutter.

Figure $2 \mathrm{a}$ shows that when a triangle wave is applied to the PZT, the comb spectrum appears twice in the time-dependent photodetector signal: first in increasing, and next in decreasing frequency order. The sequential mode filtering maps optical frequencies to the time domain with individual comb teeth arriving periodically. Such a signal is referred here to as the Vernier spectrum. The Vernier scan rate of $250 \mathrm{~Hz}$ ( $500 \mathrm{~Hz}$ for both directions) was chosen to maximize the temporal resolution while avoiding mechanical resonances and ensuring sufficient temporal separation between comb teeth (due to the cavity finesse and finite ringdown time). To confirm that the entire comb bandwidth is transmitted through the cavity, we also measured the combs's unfiltered optical spectrum with a Fourier transform spectrometer (FTIR, Bruker Vertex 80). We find good agreement between the raw emitted spectrum (Fig. 2b) and that measured by VS (plotted on the same vertical log scale in Fig. 2k). However, slight expected discrepancies are observe. First, the FTIR signal requires $1000 \times$ longer to acquire and therefore displays higher signal-to-noise ratio (SNR) than the Vernier scan. Second, the cavity introduces a lineshape convolution effect ${ }^{27}$ that ap- 

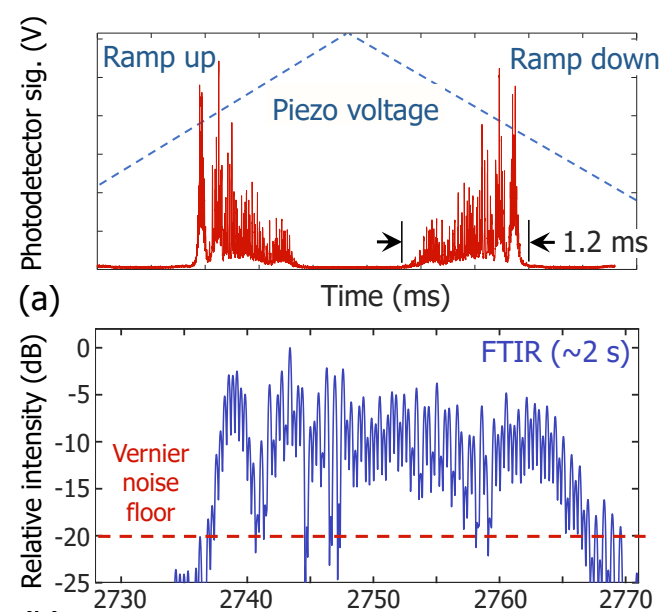

(b) Wavenumber $\left(\mathrm{cm}^{-1}\right)$

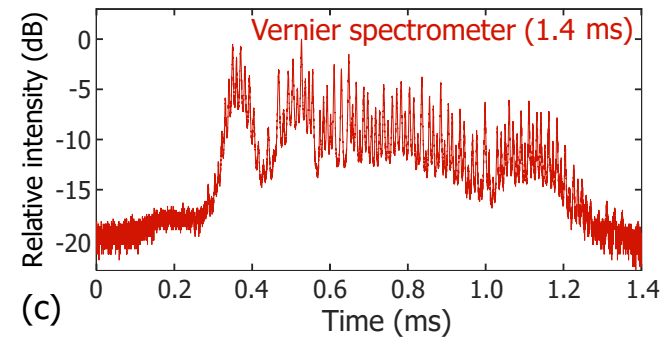

Figure 2: (a) Experimental photodetector signal (linear scale) showing both mapping directions. (b) ICL comb spectrum as measured by the FTIR. (c) Vernier spectrum plotted in log scale.

pears here as as Lorentzian-profiled lines with fullwidth at half-maximum (FWHM) $\delta_{v}=c / \Delta L \mathcal{F} \approx$ $760 \mathrm{MHz}$ (cavity filter resolution). This convolution lowers the contrast between neighboring lines, although its effect can be removed through deconvolution. In addition, the line intensities in the Vernier signal gradually decrease at higher optical frequencies (up to $2 \mathrm{~dB}$ at $2765 \mathrm{~cm}^{-1}$ ) due to progressive line broadening caused by $f_{\text {rep }}$ fluctuations that lower the comb-cavity coupling efficiency. This effect will be discussed further below.

In contrast to cavity-locked systems, the VS technique is fully compatible with free-running operation of the laser. If the laser frequency drifts, but remains relatively stable throughout the duration of a sweep ( $\sim \mathrm{MHz}$ drift), Vernier coincidence simply occurs at a different roundtrip length, although with some degradation of the Vernier spectrum. On a short timescale, dynamic optical feedback from the scanned cavity affects the ICL lasing frequency, which induces repetition-rate $f_{\text {rep }}$ and offset-frequency $f_{0}$ fluctuations. On a longer scale, laser fluctuations relative to the cavity mode frequency introduce additional frequency noise.

Time-variations of $f_{\text {rep }}$ and $f_{0}$ yield non- (a)

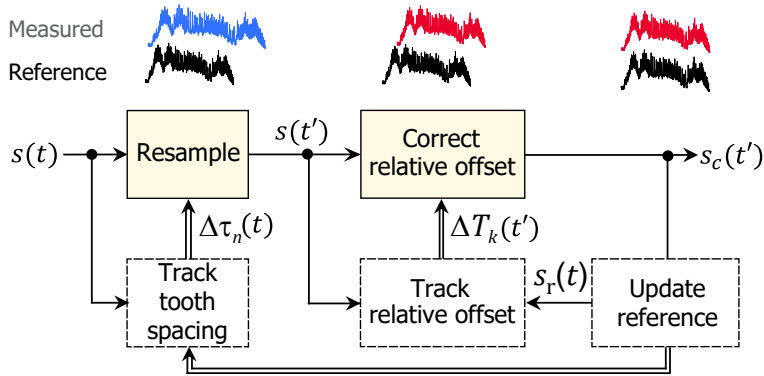

(b)

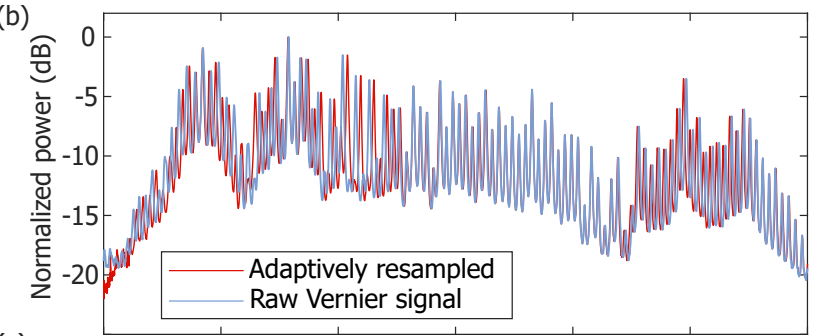

(c)

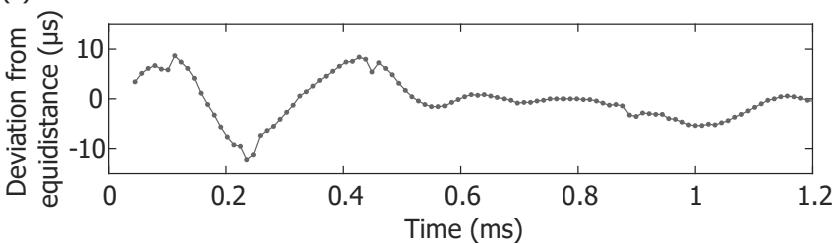

Figure 3: (a) Flowchart of the digital correction algorithm. First the time-varying spacing between the peaks is corrected through resampling. Next the relative offset with respect to the reference is eliminated via sample shift. Finally, the reference spectrum (template) is updated to permit operation under rapidly changing conditions. (b) Effect of resampling on the Vernier scan of a free-running ICL comb. (c) Instantaneous deviation from line equidistance.

equidistant peaks and shifts in the TOA of the Vernier spectrum. This complicates the spectroscopic analysis and precludes coherent averaging. To circumvent this issue, we apply a correction algorithm similar to that developed for unstabilized DCS systems, $\frac{23}{2}$ with the difference that the comb signal cannot be described by two global frequencies. Instead, the line spacing relating to $f_{\text {rep }}$ must be tracked instantaneously (line-to-line) throughout the scan (Fig. 3a). Following extraction of the spacing with a robust peak detector, the spectrum is resampled through linear interpolation onto a uniform grid. This resembles adaptive sampling as developed in the context of DCS. ${ }^{34]}$ Figure $3 \mathrm{~b}$ and 3 c plot results of the line spacing equalization. The locally compressed and expanded spectrum is then corrected through resampling.

After ensuring equidistant line spacing, we also correct for the relative time offset between the scan 
frame and reference signal (obtained from an exponential integration of the last $k$ scans/frames). Note that the down-scan must be reversed prior to alignment or simply ignored. The relative time offset $\Delta T_{k}\left(t^{\prime}\right)$ can be obtained by estimating the sample delay from the maximum of the crosscorrelation function between the current and reference frame, or by tracking a characteristic peak in a comb spectrum with a unique modal intensity pattern. Figure 4 shows the free-running VS data following averaging over 10 seconds with assistance from the digital correction algorithm. Panel (a) compares an uncorrected 200-ms-long portion (stacked 100 frames) with the full 10-s-long acquisition of 5000 spectra following correction. In the uncorrected data, the initial (low-wavenumber) portion is much sharper and shows less jitter compared to the end-of-scan part. This results from cumulative frequency shift of the $f_{\text {rep }}$ fluctuations around a fixed point. .35 The VS is found to breathe asymmetrically toward higher frequencies, with the frequency (time in the VS scan) jitter increasing uniformly with tooth number. Despite these imperfections, digital correction fully compensates the drifts. The waterfall representation in Fig. 4b illustrates that digital locking of the fluctuating VS permits power averaging.

To demonstrate an application of ICL-combbased open-path VS to real-time chemical sensing, we used our spectrometer to measure an automotive refrigerant HFC-152a - 1,1,-Difluoroethane (DFE), which was periodically released in gas phase from a nozzle located $\sim 1 \mathrm{~m}$ away from the cavity. The experiment lasting 7 seconds began with measurement of the ambient air (background) followed by quasi-periodic transient gas release events occurring at $\sim 0.8 \mathrm{~s}$ intervals starting from $t=2 \mathrm{~s}$. Figure 5 plots the results of this experiment, during which mode-resolved $1-\mathrm{THz}$-wide spectra were acquired every $2 \mathrm{~ms}$. After digital scan alignment, we calibrated the optical frequency axis based on the known $f_{\text {rep }}$ and emission wavelength range. To illustrate the temporal averaging capabilities, Fig. 5 a plots spectra from a later stage of the experiment during quasi-constant DFE concentration. The semi-transparent grey points show modal intensities during a single scan $(2 \mathrm{~ms})$ of the dynamicallyevolving signal, while the blue-scale points were acquired with longer averaging (100 ms) over 50 scans. The measured spectra show good agreement with HITRAN data ${ }^{36}$ corresponding to a 2600 ppm fit of DFE (red curve).
We see in Fig. 5at that some line intensities fluctuate more than others, which depends on the differing powers emitted in corresponding teeth of the ICL comb [see Fig. 2k]. To visualize the nonheteroskedacity (differing precisions) of the data, the fills of the averaged modal intensity points are coloured based on the relative line strength with stronger lines represented by a darker blue. The darker points naturally offer greater precision and deviate less from the model. Nevertheless, a wavy etalon-like structure is superimposed, which is attributable to parasitic reflections at optical interfaces in the system. To systematically characterize the spectroscopic precision attainable for all comb teeth, Fig. 5b illustrates the standard deviation of the ratio of consecutive background spectra without the analyte. The standard deviation $s$ of $4.3 \%$ at $300 \mathrm{~ms}$ is dominated by outliers with weaker modal intensities. This indicates the need to equalize the spectral envelope of future ICL combs.

Figure 5. plots the time-resolved optical spectra acquired in the dynamic gas release experiment. As in Fig. 5 a, it shows intensive broadband absorption caused by the DFE at $2755 \mathrm{~cm}^{-1}$ along with a weaker feature at $2742 \mathrm{~cm}^{-1}$. In the scanned region, the DFE absorption cross-section reaches $\sim 10^{-21} \mathrm{~cm}^{2} /$ molecule. Although this is two orders of magnitude weaker than at optimal mid-IR wavelengths, it is nonetheless sufficient to demonstrate ppm-level transient detection of the molecule. Figure $5 \mathrm{~d}$ plots the fitted DFE concentration as a function of time, which can be visually compared with modal intesities at 10 arbitrary comb frequencies in Fig. 5e. From the background obtained in the first two seconds, we calculate the standard deviation for concentration $s_{\mathrm{b}}=43.5 \mathrm{ppm}$. Then at $t_{0}=2$, we see a rapid increase to the $1000 \mathrm{~s}$ of ppm range, followed by a slow decay as the released gas diffuses in the ambient air (with some oscillations due to turbulent flow of the gas released under pressure). The final $5 \mathrm{~s}$ of the scan captures six release events with similar profiles. From the one occurring before $t=4 \mathrm{~s}$, we retrieve a quasisteady state concentration of 1000 ppm with standard deviation $s=72.2 \mathrm{ppm}$ at $2 \mathrm{~ms}$ timescale (framed part of Fig. 5d). The discrepancy between this value $(s)$ and that retrieved at the beginning of the experiment $\left(s_{\mathrm{b}}\right)$ results from the Rice distribution of the data. At zero gas concentration, the noise is underestimated because the concentration cannot be negative and hence deviates less from the true mean $(0)$. The underestimation factor 37 

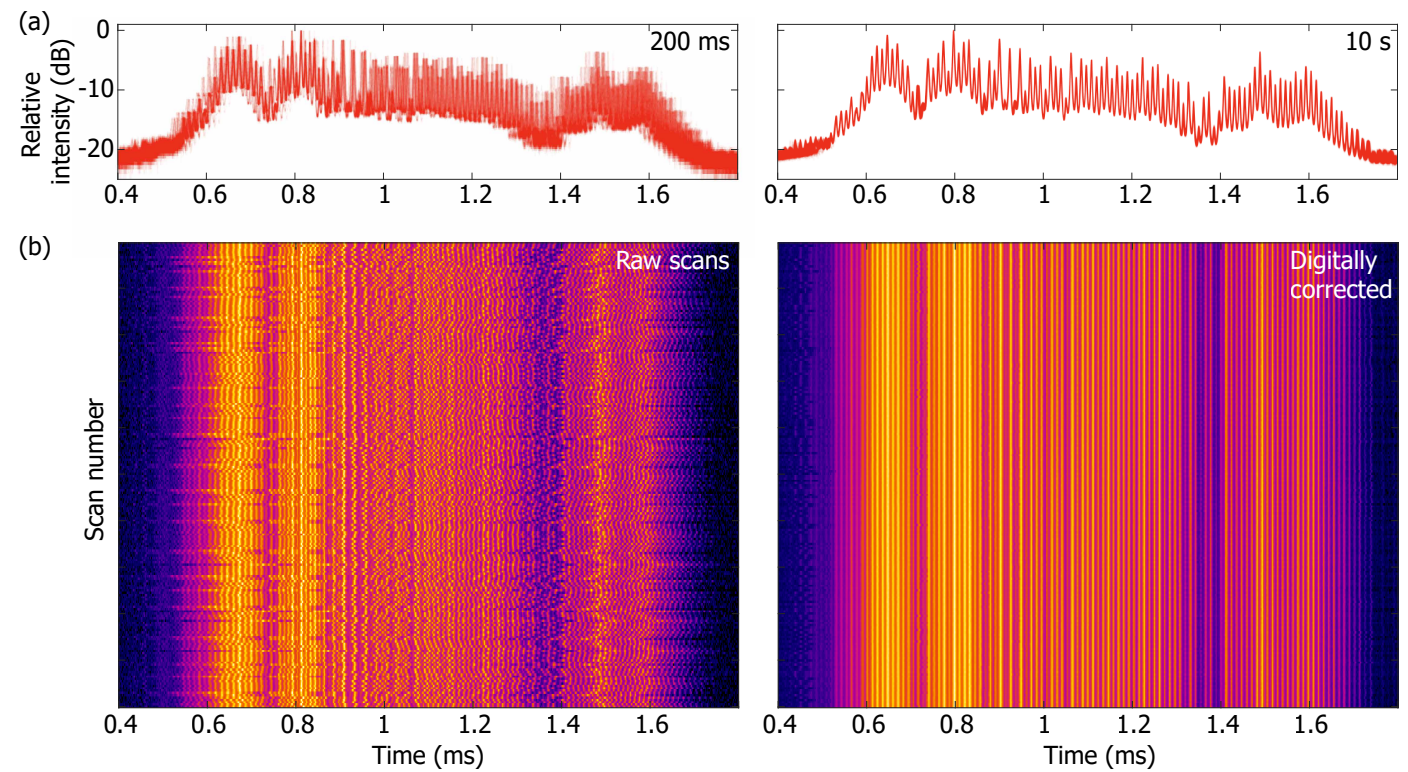

Figure 4: (a) Stacked $200 \mathrm{~ms}$ of uncorrected Vernier scans (2 ms each, left), compared to a full $10 \mathrm{~s}$ acquisition following digital correction (right). (b) Waterfall plot of the data in (a), revealing shifts of the peak positions that are compensated by the algorithm.
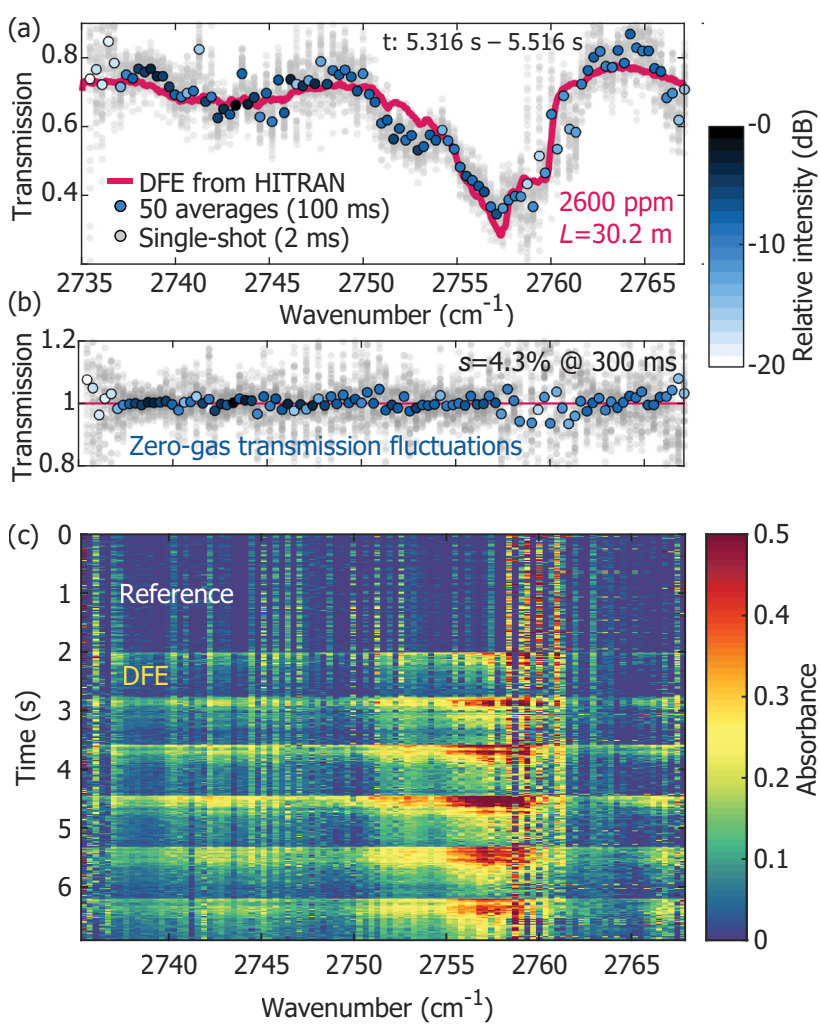

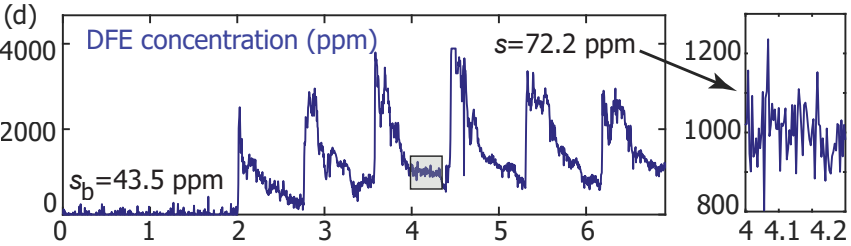

(e)

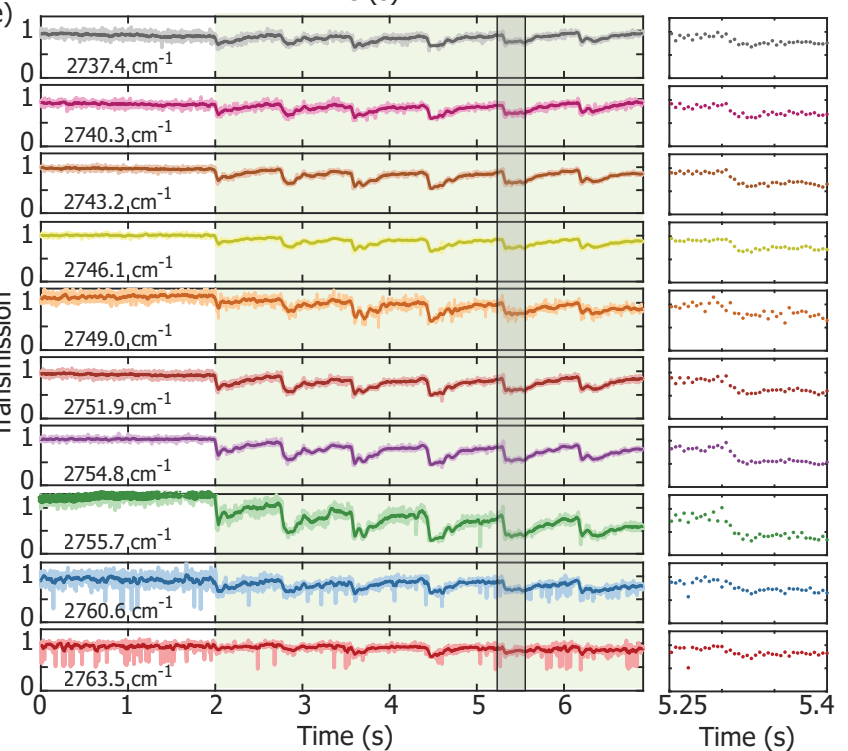

Figure 5: (a) VS transmission spectrum (points) along with results from a HITRAN 2016 absorption cross-section model ${ }^{36}$ of DFE (red curve). The semi-transparent grey points are single measurements retrieved from the dynamically-evolving signal, while the blue-scale points are averaged over 50 scans for higher precision [darker $=$ higher]. (b) Zero-gas (reference) measurement that characterizes the standard deviation of scan-to-scan transmission fluctuations. (c) Spectrogram of the DFE experiment. The vertical features around $2760 \mathrm{~cm}^{-1}$, which also appear in the reference spectra, are due to the low-SNR of the relevant comb lines. (d) Time dependenence of the fitted DFE concentration. (e) Transmission of select comb teeth with a zoom on one of the transient absorption events. 


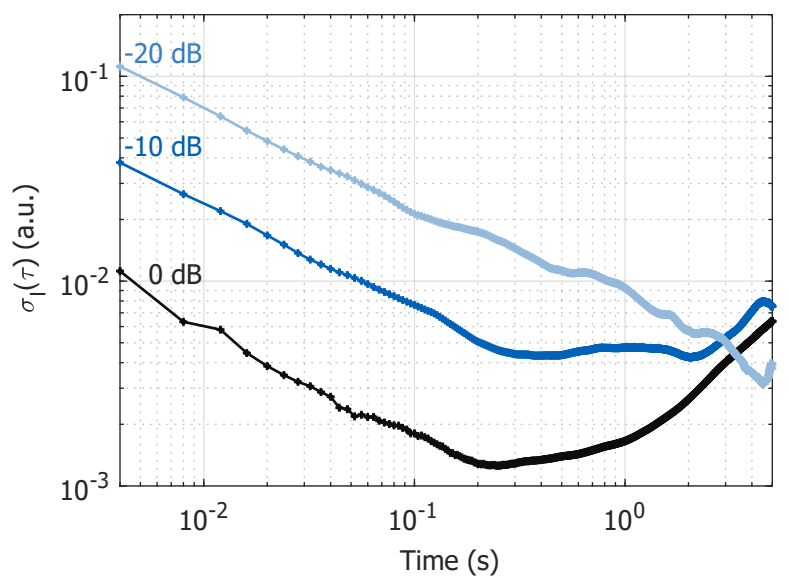

Figure 6: Allan deviation plot of the relative intensities for comb lines with three different power levels. The strongest line reaches a minimum in the per-mille precision region, followed by drift after $200 \mathrm{~ms}$.

$\sqrt{2-\pi / 2} \approx 0.655$ renders the value $\hat{s} \approx 66 \mathrm{ppm}$, which is close to the result $72.2 \mathrm{ppm}$ measured with the analyte. Note that at $2 \mathrm{~ms}$, an ICL comb operating at the near-optimal wavelength $(\lambda \approx 3.36 \mu \mathrm{m})$ for DFE may be expected to yield a sub-ppm instantaneous detection limit $(1 \sigma)$.

To fully characterize the system's capability for averaging comb line intensities, we performed an Allan-Werle deviation analysis. $\frac{38}{}$ For this we chose the strongest comb line, along with two arbitrary comb lines that were weaker by $10 \mathrm{~dB}$, and $20 \mathrm{~dB}$. These three lines at $\nu_{0}=2744 \mathrm{~cm}^{-1}, \nu_{-10}=$ $2762 \mathrm{~cm}^{-1}$, and $\nu_{-20}=2735 \mathrm{~cm}^{-1}$ were used to generate Fig. 6. As expected, for ms timescales a $20 \mathrm{~dB}$ decrease in intensity yields $10 \times$ loss in precision. At $2 \mathrm{~ms}$, the precision of the strongest line is already $\sim 1 \%$, whereas that for the weakest is $10 \%$. At sub-second timescales, the $0-\mathrm{dB}$ and $-10-\mathrm{dB}$ curves eventually reach a plateau followed by drift, whereas the $-20-\mathrm{dB}$ result (dominated by whitenoise) continues to improve up to $5 \mathrm{~s}$. A reasonable integration time can be derived from the approximate intercept of all three curves at $\sigma_{I} \approx 0.5 \%$, which occurs at $\tau \approx 3 \mathrm{~s}$. The differing trends of the three curves may be attributed to their corresponding levels of white noise. For instance, the $-10-\mathrm{dB}$ line reaches a plateau when the $0-\mathrm{dB}$ line is already in drift because its averaged noise and drift terms cancel at that point. A more rapid increase of the drift (after $2 \mathrm{~s}$ ) breaks the plateau balance and causes the $-10-\mathrm{dB}$ curve to increase as well. The averaging capability is also influenced imperfections in the peak alignment algorithm.
The best-case minimum detectable absorption (MDA) $\sigma_{\min }$ estimated for the strongest line is $1.3 \times 10^{-3}$ at $\tau=240 \mathrm{~ms}$, which corresponds to a noise-equivalent absorption (NEA) of $\sigma \tau^{1 / 2}=$ $6.4 \times 10^{-4} \mathrm{~Hz}^{-1 / 2}$, and noise equivalent absorption coefficient $(\mathrm{NEAC})$ of $\mathrm{NEA} / L_{\mathrm{eff}}=2.1 \times$ $10^{-7} \mathrm{~cm}^{-1} \mathrm{~Hz}^{-1}$. We emphasize, however, that these numbers do not reflect the broadband sensing capabilities of the demonstrated VS system. We can instead use the background standard deviation of $4.3 \%$ at $300 \mathrm{~ms}$, from which the full-bandwidth $\mathrm{NEA}$ is $2.4 \times 10^{-2} \mathrm{~Hz}^{-1 / 2}$, and the NEAC is $7.8 \times 10^{-6} \mathrm{~cm}^{-1} \mathrm{~Hz}^{-1}$. While this is two orders of magnitude lower than for a state-of-the-art VS based on stabilized mid-IR optical parametric oscillators (OPO) on a table top, $\underline{29}$ the ICL-combbased system combines the advantages of compactness, simplicity, mode resolution and free-running operation. Besides tuning the comb spectrum to a near-optimal wavelength $\lambda \approx 3.36 \mu \mathrm{m}$, future systems with benefit from improved modal structure that provides a more uniform optical power distribution without spectral gaps. $\frac{12}{12}$

This mode-resolved VS technique opens exciting possibilities for sensitive and robust in-situ detection in field campaigns or as a benchtop instrument. Drastically reducing the overall size from this initial demonstration will be straightforward given the limited number of components and the compactness of the ICL comb. Ultimately, all of the optics and the laser could fit within $10 \mathrm{~cm}$ without any degradation in performance. This is much smaller than what is possible for FTIR instruments, and is competitive with miniature dual-comb spectrometers.

Future work can further enhance the stability and sensing sensitivity by increasing the finesse or length of the optical cavity. Alternative detection schemes, like cavity ring-down spectroscopy 39 one-dimensional spectroscopy, 40 can also be implemented using the same experimental setup. The spectral resolution can be improved by tuning the output spectrum via the injection current, so as to fill spectral gaps in the coarse sampling grid defined by the comb's repetition rate. ${ }^{41}$ Note from Figs. 2(b) and 5(a) that under the default operating conditions of the demonstration, the DFE absorption spectrum spanned a region of weak comb emission near $2758 \mathrm{~cm}^{-1}$.

While the dual-comb technique is a popular choice for many comb-based spectrometers, moderesolved Vernier spectroscopy may be viewed as 
complementary. Its optical path enhancement and selective line filtering eases the requirement for a fast photodetector, microwave digitizers, and two matched comb sources. Despite the lack of truly multiplexed multi-line detection for all the modes simultaneously and constraints on the temporal resolution, advantages include the technique's compatibility with both free-running comb operation and cavity enhancement. These two features allow a relatively simple setup to reach high spectroscopic sensitivity.

In conclusion, a chip-scale mid-IR interband cascade laser frequency comb has been incorporated into a Vernier spectrometer in which the optical cavity enhances the path length, while also selectively filtering the individual comb lines. The ICL comb source with $\mathrm{GHz}$ repetition rate and $1 \mathrm{THz}$ of optical bandwidth is well suited to the VS configuration. Under free-running operation in a cavity only $3 \mathrm{~cm}$ long, the spectrometer reaches ppm-level sensitivity. The VS also allows dynamic sensing with temporal resolution limited primarily by the cavity scanning rate. With an open-cavity cell, we demonstrate broadband detection of a hydrocarbon in real time with millisecond refresh rate. This prototype system shows the promise of future miniaturized cavity-enhanced spectrometers for chemical sensing. Moreover, the concept may be extended to semiconductor microcombs spanning other spectral regions e.g. QCL combs operating in the long-wave infrared or THz.

\section{Notes}

The authors declare no conflicts of interest.

Acknowledgement This work was supported under and was in part performed at the Jet Propulsion Laboratory (JPL), California Institute of Technology, under contract with NASA. L. A. Sterczewski's research was supported by an appointment to the NASA Postdoctoral Program at JPL, administered by Universities Space Research Association under contract with NASA. The NRL authors acknowledge support from the Office of Naval Research (ONR). C. R. M. is grateful for support from the Arnold and Mabel Beckman Foundation through the A. O. Beckman Postdoctoral Fellowship.

Funding. National Aeronautics and Space Agency's (NASA) PICASSO program (106822 / 811073.02.24.01.85), PDRDF program; Universi- ties Space Research Association (USRA), NASA Postdoctoral Program (NPP).

\section{References}

(1) Picqué, N.; Hänsch, T. W. Frequency Comb Spectroscopy. Nature Photonics 2019, 13, 146-157.

(2) Fortier, T.; Baumann, E. 20 years of developments in optical frequency comb technology and applications. Communications Physics 2019, 2, 1-16.

(3) Spaun, B.; Changala, P. B.; Patterson, D.; Bjork, B. J.; Heckl, O. H.; Doyle, J. M.; Ye, J. Continuous probing of cold complex molecules with infrared frequency comb spectroscopy. Nature 2016, 533, 517-520.

(4) Hänsch, T. W. Nobel Lecture: Passion for Precision. Reviews of Modern Physics 2006, 78, 1297-1309.

(5) Bjork, B. J.; Bui, T. Q.; Heckl, O. H.; Changala, P. B.; Spaun, B.; Heu, P.; Follman, D.; Deutsch, C.; Cole, G. D.; Aspelmeyer, M.; Okumura, M.; Ye, J. Direct frequency comb measurement of $\mathrm{OD}+\mathrm{CO} \rightarrow$ DOCO kinetics. Science 2016, 354, 444-448.

(6) Pasquazi, A.; Peccianti, M.; Razzari, L.; Moss, D. J.; Coen, S.; Erkintalo, M.; Chembo, Y. K.; Hansson, T.; Wabnitz, S.; Del'Haye, P.; Xue, X.; Weiner, A. M.; Morandotti, R. Micro-Combs: A Novel Generation of Optical Sources. Physics Reports 2018, 729, 1-81.

(7) Scalari, G.; Faist, J.; Picqué, N. On-Chip MidInfrared and $\mathrm{THz}$ Frequency Combs for Spectroscopy. Applied Physics Letters 2019, 114, 150401.

(8) Bao, C.; Yuan, Z.; Wang, H.; Wu, L.; Shen, B.; Sung, K.; Leifer, S.; Lin, Q.; Vahala, K. Interleaved difference-frequency generation for microcomb spectral densification in the mid-infrared. Optica 2020, 7, 309-315.

(9) Hugi, A.; Villares, G.; Blaser, S.; Liu, H.; Faist, J. Mid-infrared frequency comb based on a quantum cascade laser. Nature 2012, 492, 229-233. 
(10) Bagheri, M.; Frez, C.; Sterczewski, L. A.; Gruidin, I.; Fradet, M.; Vurgaftman, I.; Canedy, C. L.; Bewley, W. W.; Merritt, C. D.; Kim, C. S.; Kim, M.; Meyer, J. R. Passively Mode-Locked Interband Cascade Optical Frequency Combs. Scientific Reports 2018, 8, 3322.

(11) Schwarz, B.; Hillbrand, J.; Beiser, M.; Andrews, A. M.; Strasser, G.; Detz, H.; Schade, A.; Weih, R.; Höfling, S. Monolithic frequency comb platform based on interband cascade lasers and detectors. Optica 2019, 6, 890-895.

(12) Sterczewski, L. A.; Bagheri, M.; Frez, C.; Canedy, C. L.; Vurgaftman, I.; Kim, M.; Kim, C. S.; Merritt, C. D.; Bewley, W. W.; Meyer, J. R. Interband cascade laser frequency combs. Journal of Physics: Photonics 2021, 3, 042003.

(13) Kawai, A.; Hashimoto, K.; Dougakiuchi, T.; Badarla, V. R.; Imamura, T.; Edamura, T.; Ideguchi, T. Time-stretch infrared spectroscopy. Communications Physics 2020, 3, $1-6$.

(14) Coddington, I.; Newbury, N.; Swann, W. Dual-Comb Spectroscopy. Optica 2016, 3, 414.

(15) Sterczewski, L. A.; Westberg, J.; Yang, Y.; Burghoff, D.; Reno, J.; Hu, Q.; Wysocki, G. Terahertz Spectroscopy of Gas Mixtures with Dual Quantum Cascade Laser Frequency Combs. ACS Photonics 2020, 7, 1082-1087.

(16) Sterczewski, L. A.; Westberg, J.; Bagheri, M.; Frez, C.; Vurgaftman, I.; Canedy, C. L.; Bewley, W. W.; Merritt, C. D.; Kim, C. S.; Kim, M.; Meyer, J. R.; Wysocki, G. MidInfrared Dual-Comb Spectroscopy with Interband Cascade Lasers. Optics Letters 2019, 44, 2113-2116.

(17) Klocke, J. L.; Mangold, M.; Allmendinger, P.; Hugi, A.; Geiser, M.; Jouy, P.; Faist, J.; Kottke, T. Single-Shot Sub-Microsecond MidInfrared Spectroscopy on Protein Reactions with Quantum Cascade Laser Frequency Combs. Analytical Chemistry 2018, 90, 10494-10500.
(18) Luo, P.-L. Long-wave mid-infrared timeresolved dual-comb spectroscopy of shortlived intermediates. Optics Letters 2020, 45, 6791-6794.

(19) Bernhardt, B.; Ozawa, A.; Jacquet, P.; Jacquey, M.; Kobayashi, Y.; Udem, T.; Holzwarth, R.; Guelachvili, G.; Hänsch, T. W.; Picqué, N. Cavity-enhanced dual-comb spectroscopy. Nature Photonics 2010, 4, 55-57.

(20) Hoghooghi, N.; Wright, R. J.; Makowiecki, A. S.; Swann, W. C.; Waxman, E. M.; Coddington, I.; Rieker, G. B. Broadband coherent cavity-enhanced dualcomb spectroscopy. Optica 2019, 6, 28-33.

(21) Liao, R.; Tian, H.; Liu, W.; Li, R.; Song, Y.; $\mathrm{Hu}, \mathrm{M}$. Dual-Comb Generation from a Single Laser Source: Principles and Spectroscopic Applications towards Mid-IR - A Review. Journal of Physics: Photonics 2020, 2, 042006.

(22) Burghoff, D.; Yang, Y.; Hu, Q. Computational Multiheterodyne Spectroscopy. Science Advances 2016, 2, e1601227.

(23) Sterczewski, L. A.; Westberg, J.; Wysocki, G. Computational Coherent Averaging for FreeRunning Dual-Comb Spectroscopy. Optics Express 2019, 27, 23875-23893.

(24) Sterczewski, Ł. A.; Przewłoka, A.; Kaszub, W.; Sotor, J. Computational Doppler-Limited Dual-Comb Spectroscopy with a Free-Running All-Fiber Laser. APL Photonics 2019, 4, 116102.

(25) Gohle, C.; Stein, B.; Schliesser, A.; Udem, T.; Hänsch, T. W. Frequency Comb Vernier Spectroscopy for Broadband, High-Resolution, High-Sensitivity Absorption and Dispersion Spectra. Physical Review Letters 2007, 99, 263902.

(26) Vernier, P. La construction, l'usage et les propriétez du quadrant nouveau de mathématique; Fr. Vivien., 1631.

(27) Rutkowski, L.; Morville, J. Broadband Cavity-Enhanced Molecular Spectra from Vernier Filtering of a Complete Frequency Comb. Optics Letters 2014, 39, 6664-6667. 
(28) Khodabakhsh, A.; Ramaiah-Badarla, V.; Rutkowski, L.; Johansson, A. C.; Lee, K. F.; Jiang, J.; Mohr, C.; Fermann, M. E.; Foltynowicz, A. Fourier Transform and Vernier Spectroscopy Using an Optical Frequency Comb at 3-5.4 $\mu \mathrm{m}$. Optics Letters 2016, 41, 2541.

(29) Khodabakhsh, A.; Rutkowski, L.; Morville, J.; Foltynowicz, A. Mid-Infrared ContinuousFiltering Vernier Spectroscopy Using a Doubly Resonant Optical Parametric Oscillator. Applied Physics B 2017, 123, 210.

(30) Nugent-Glandorf, L.; Neely, T.; Adler, F.; Fleisher, A. J.; Cossel, K. C.; Bjork, B.; Dinneen, T.; Ye, J.; Diddams, S. A. Mid-Infrared Virtually Imaged Phased Array Spectrometer for Rapid and Broadband Trace Gas Detection. Optics Letters 2012, 37, 3285.

(31) Hindle, F.; Bocquet, R.; Pienkina, A.; Cuisset, A.; Mouret, G. Terahertz Gas Phase Spectroscopy Using a High-Finesse Fabry-Pérot Cavity. Optica 2019, 6, 1449.

(32) Meyer, J. R.; Bewley, W. W.; Canedy, C. L.; Kim, C. S.; Kim, M.; Merritt, C. D.; Vurgaftman, I. The Interband Cascade Laser. Photonics 2020, 7, 75, Issue: 3.

(33) Sterczewski, L. A.; Bagheri, M.; Frez, C.; Canedy, C. L.; Vurgaftman, I.; Meyer, J. R. Mid-Infrared Dual-Comb Spectroscopy with Room-Temperature Bi-Functional Interband Cascade Lasers and Detectors. Applied Physics Letters 2020, 116, 141102.

(34) Ideguchi, T.; Poisson, A.; Guelachvili, G.; Picqué, N.; Hänsch, T. W. Adaptive real-time dual-comb spectroscopy. Nature Communications 2014, 5, 1-8.

(35) Newbury, N. R.; Swann, W. C. Low-noise fiber-laser frequency combs (Invited). Journal of the Optical Society of America B 2007, 24, 1756.

(36) Kochanov, R. V.; Gordon, I. E.; Rothman, L. S.; Shine, K. P.; Sharpe, S. W.; Johnson, T. J.; Wallington, T. J.; Harrison, J. J.; Bernath, P. F.; Birk, M.; Wagner, G.; Le Bris, K.; Bravo, I.; Hill, C. Infrared Absorption Cross-Sections in HITRAN2016 and beyond: Expansion for Climate, Environment, and Atmospheric Applications. Journal of Quantitative Spectroscopy and Radiative Transfer 2019, 230, 172-221.

(37) Gudbjartsson, H.; Patz, S. The Rician distribution of noisy MRI data. Magnetic Resonance in Medicine 1995, 34, 910-914.

(38) Werle, P.; Mücke, R.; Slemr, F. The limits of signal averaging in atmospheric tracegas monitoring by tunable diode-laser absorption spectroscopy (TDLAS). Applied Physics $B$ 1993, 57, 131-139.

(39) Orr, B. J.; He, Y. Rapidly swept continuouswave cavity-ringdown spectroscopy. Chemical Physics Letters 2011, 512, 1-20.

(40) Cygan, A.; Wcisło, P.; Wójtewicz, S.; Masłowski, P.; Hodges, J. T.; Ciuryło, R.; Lisak, D. One-dimensional frequency-based spectroscopy. Optics Express 2015, 23, 14472-14486.

(41) Gianella, M.; Nataraj, A.; Tuzson, B.; Jouy, P.; Kapsalidis, F.; Beck, M.; Mangold, M.; Hugi, A.; Faist, J.; Emmenegger, L. High-resolution and gapless dual comb spectroscopy with current-tuned quantum cascade lasers. Optics Express 2020, 28, 6197-6208. 